\title{
Filtering and Mining Parallel Data in a Joint Multilingual Space
}

\author{
Holger Schwenk \\ Facebook AI Research \\ schwenk@dfb.com
}

\begin{abstract}
We learn a joint multilingual sentence embedding and use the distance between sentences in different languages to filter noisy parallel data and to mine for parallel data in large news collections. We are able to improve a competitive baseline on the WMT'14 English to German task by 0.3 BLEU by filtering out $25 \%$ of the training data. The same approach is used to mine additional bitexts for the WMT'14 system and to obtain competitive results on the BUCC shared task to identify parallel sentences in comparable corpora.

The approach is generic, it can be applied to many language pairs and it is independent of the architecture of the machine translation system.
\end{abstract}

\section{Introduction}

Parallel data, also called bitexts, is an important resource to train neural machine translation systems (NMT). It is usually assumed that the quality of the automatic translations increases with the amount of available training data. However, it was observed that NMT systems are more sensitive to noise than SMT systems, e.g. (Belinkov and Bisk, 2017). Well known sources of parallel data are international organizations like the European Parliament or the United Nations, or community provided translations like the TED talks. In addition, there are many texts on the Internet which are potential mutual translations, but which need to be identified and aligned. Typical examples are Wikipedia or news collections which report on the same facts in different languages. These collections are usually called comparable corpora.

In this paper we propose an unified approach to filter noisy bitexts and to mine bitexts in huge monolingual texts. The main idea is to first learn a joint multilingual sentence embedding. Then, a threshold on the distance between two sentences in this joint embedding space can be used to filter bitexts (distance between source and target sentences), or to mine for additional bitexts (pairwise distances between all source and target sentences). No additional features or classifiers are needed.

\section{Related work}

The problem of how to select parts of bitexts has been addressed before, but mainly from the aspect of domain adaptation (Axelrod et al., 2011; Santamaría and Axelrod, 2017). It was successfully used in many phrase-based MT systems, but it was reported to be less successful for NMT (van der Wees et al., 2017). It should be stressed that domain adaptation is different from filtering noisy training data. Data selection extracts the most relevant bitexts for the test set domain, but does not necessarily remove wrong translations, e.g. source and target sentences are both in-domain and well formed, but they are not mutual translations.

There is a huge body of research on mining bitexts, e.g. by analyzing the name of WEB pages or links (Resnik and Smith, 2003). Another direction of research is to use cross-lingual information retrieval, e.g. (Utiyama and Isahara, 2003; Munteanu and Marcu, 2005; Rauf and Schwenk, 2009). There are some works which use joint embeddings in the process of filtering or mining bitexts. For instance, Grégoire and Langlais (2017) first embed sentences into two separate spaces. Then, a classifier is learned on labeled data to decide whether sentences are parallel or not. Our approach clearly outperforms this technique on the BUCC corpus (cf. section 4). Bouamor and Sajjad (2018) use averaged multilingual word embeddings to calculate a joint embedding of all sen- 
tences. However, distances between all sentences are only used to extract a set of potential mutual translation. The decision is based on a different system. In Hassan et al. (2018) NMT systems for $\mathrm{Zh} \leftrightarrow \mathrm{En}$ are learned using a joint encoder. A sentence representation is obtained as the mean of the last encoder states. Noisy bitexts are filtered based on the distance. In all these works, embeddings are learned for two languages only, while we learn one joint embedding for up to nine languages.

\section{Multilingual sentence embeddings}

We are aiming at an embedding of entire sentences in different languages into one joint space, with the goal that the distance in that space reflects their semantic difference, independently of the language. There are several works on learning multilingual sentence embeddings which could be used for that purpose, i.e. (Hermann and Blunsom, 2014; Pham et al., 2015; Zhou et al., 2016; Chandar et al., 2013; Mogadala and Rettinger, 2016).

In this paper, we extend our initial approach (Schwenk and Douze, 2017). The underlying idea is to use multiple sequence encoders and decoders and to train them with $N$-way aligned corpora from the MT community. Instead of using one encoder for each language as in the original paper, we use a shared encoder which handles all the input languages. Joint encoders (and decoders) have already been used in NMT (Johnson et al., 2016). In contrast to that work, we do not use a special input token to indicate the target language. Our joint encoder has no information at all on the encoded language, or what will be done with sentence representation.

We trained this architecture on nine languages ${ }^{1}$ of the Europarl corpus with about 2M sentences each. We use BPE (Sennrich et al., 2016b) to learn one 20k joint vocabulary for all the nine languages. $^{2}$ The joint encoder is a 3-layer BLSTM. The word embeddings are of size 384 and the hidden layer of the BLSTM is 512-dimensional. The 1024 dimensional sentence embedding is obtained by max-pooling over the BLSTM outputs. Dropout is set to 0.1 . These settings are identical to those reported in (Schwenk and Douze, 2017), with the difference that we observe slight improvement by using a deeper network for the joint encoder. Once the system is learned, all the BLSTM

\footnotetext{
${ }^{1}$ en, fr, es, it, pt, de, da, nl and fi

${ }^{2}$ Larger vocabularies achieve only slight improvements.
}

decoders are discarded and we only use the multilingual BLSTM encoder to embed the sentences into the joint space.

A very similar approach was also proposed in España-Bonet et al. (2017). A joint NMT system with attention is trained on several languages pairs, similar to (Johnson et al., 2016), including a special token to indicate the target language. After training, the sum of the encoder output states is used to obtain a fixed size sentence representation.

\section{Experimental evaluation: BUCC shared task on mining bitexts}

Since 2017, the workshop on Building and Using Comparable Corpora (BUCC) is organizing a shared task to evaluate the performance of approaches to mine for parallel sentences in comparable corpora (Zweigenbaum et al., 2018). Table 1 summarizes the available data, and Table 2 the official results. Roughly a 40th of the sentences are aligned. The best performing system "VIC" is based on the so-called STACC method which was shown to achieve state-of-the-art performance (Etchegoyhen and Azpeitia, 2016). It combines probabilistic dictionaries, search for similar sentences in both directions and a decision module which explores various features (common word prefixes, numbers, capitalized true-case tokens, etc). This STACC system was improved and adapted to the BUCC tasks with a word weighting scheme which is optimized on the monolingual corpora, and a named entity penalty. This task adaption substantially improved the generic STACC approach (Azpeitia et al., 2018). The systems RALI (Grégoire and Langlais, 2017) and H2 (Bouamor and Sajjad, 2018) have been already described in section 2. NLP2CT uses a denoising auto-encoder and a maximum-entropy classifier (Leong et al., 2018).

We applied our approach to all language pairs of the BUCC shared task (see Table 3). We used the

\begin{tabular}{|l|rrr|rr|}
\hline Lang. & \multicolumn{3}{|c|}{ Train } & \multicolumn{2}{c|}{ Test } \\
Pair & en & other & aligned & en & other \\
\hline en-de & $400 \mathrm{k}$ & $414 \mathrm{k}$ & 9580 & $397 \mathrm{k}$ & $414 \mathrm{k}$ \\
en-fr & $370 \mathrm{k}$ & $272 \mathrm{k}$ & 9086 & $373 \mathrm{k}$ & $277 \mathrm{k}$ \\
en-ru & $558 \mathrm{k}$ & $461 \mathrm{k}$ & 14435 & $566 \mathrm{k}$ & $457 \mathrm{k}$ \\
en-zh & $89 \mathrm{k}$ & $95 \mathrm{k}$ & 1899 & $90 \mathrm{k}$ & $92 \mathrm{k}$ \\
\hline
\end{tabular}

Table 1: BUCC evaluation to mine bitexts. Number of sentences and size of the gold alignments. 


\begin{tabular}{|l|c|c|c|c|}
\hline System & en-fr & en-de & en-ru & en-zh \\
\hline VIC'17 & 79 & 84 & - & - \\
RALI'17 & 20 & - & - & - \\
LIMSI'17 & - & - & - & 43 \\
\hline VIC'18 & 81 & 86 & 81 & 77 \\
H2'18 & 76 & - & - & - \\
NLP2CT'18 & - & - & - & 56 \\
\hline \hline
\end{tabular}

Table 2: Official test set results of the 2017 and 2018 BUCC shared tasks (F-scores).

embeddings from (Schwenk and Douze, 2017) for ru and zh, which were trained on the UN corpus. The only task-specific adaptation is the optimization of the threshold on the distance in the multilingual joint space. Our system does not match the performance of the heavily tuned VIC system, but it is on-pair with $\mathrm{H} 2$ on en-fr, and outperforms all other approaches by a large margin. We would like to emphasize that our approach uses no additional features or classifiers, and that we apply the same approach to all language pairs. It is nice to see that the performance varies little for the languages.

España-Bonet et al. (2017) have also evaluated their technique on the BUCC data, but results on the official test set are not provided. Also, their joint encoder uses the "news-commentary" corpus during training. This is likely to add an important bias since all the parallel sentences in the BUCC corpus are from the news-commentary corpus.

Since we learn multilingual embeddings for many languages in one joint space, we can mine for parallel data for any language pair. As an example, we have mined for French/German and Chinese/Russian bitexts, respectively. There are no reference alignments to optimize the threshold for this language pair. Based on the experiments with the other languages, we chose a value of 0.55 .

\begin{tabular}{|c|c|c|c|c|c|}
\hline \multicolumn{2}{|l|}{ Task } & en-fr & en-de & en-ru & en-zh \\
\hline \multirow{3}{*}{ Train } & $\mathrm{P}$ & 81.9 & 82.2 & 79.9 & 76.7 \\
\hline & $\mathrm{R}$ & 69.1 & 70.1 & 67.8 & 67.1 \\
\hline & $\mathrm{F} 1$ & 74.9 & 76.1 & 73.3 & 71.6 \\
\hline \multicolumn{2}{|c|}{ Threshold } & 0.58 & 0.50 & 0.57 & 0.64 \\
\hline \multirow{3}{*}{ Test } & $\mathrm{P}$ & 84.8 & 84.1 & 81.1 & 77.7 \\
\hline & $\mathrm{R}$ & 68.6 & 70.7 & 67.6 & 66.4 \\
\hline & F1 & 75.8 & 76.9 & 73.8 & 71.6 \\
\hline
\end{tabular}

Table 3: Results on the BUCC test set of our approach: Precision, Recall and F-measure (\%). We also provide the optimal threshold.
In the annex, we provide examples of extracted parallel sentences for various values of the multilingual distance. These examples show that our approach may wrongly align sentences which are mainly an enumeration of named entities, numerical values, etc. Many of these erroneous alignments could be possibly excluded by some postprocessing, e.g. comparing the number of named entities in each sentence.

\section{Experimental evaluation: improving WMT'14 En-De NMT systems}

\subsection{Baseline NMT systems}

We have performed all our experiments with the freely available Sequence-to-Sequence PyTorch toolkit from Facebook AI Research, ${ }^{3}$ called fairseq-py. It implements a convolutional model which achieves very competitive results (Gehring et al., 2017). We use this system to show the improvements obtained by filtering the standard training data and by integrating additional mined data. We will freely share this data so that it can be used to train different NMT architectures.

In this work, we focus on translating from English into German using the WMT'14 data. This task was selected for two reasons:

- it is the de-facto standard to evaluate NMT systems and many comparable results are available, e.g. (Sennrich et al., 2016b; Chunga et al., 2016; Wu et al., 2016; Gehring et al., 2017; Ashish Vaswani et al., 2017);

- only a limited amount of parallel training data is available (4.5M sentences). $2.1 \mathrm{M}$ are high quality human translations and $2.4 \mathrm{M}$ are crawled and aligned sentences (Common Crawl corpus).

As in other works, we use newstest-2014 as test set. However, in order to follow the standard WMT evaluation setting, we use mteval-v14.pl on untokenized hypothesis to calculate case-sensitive BLEU scores. Note that in some papers, BLEU is calculated with multi-bleu.perl on tokenized hypothesis. All our results are for one single system only.

We trained the fairseq-py system with default parameters, but a slightly different pre- and

\footnotetext{
${ }^{3}$ https: //github.com/facebookresearch/ fairseq-py
} 


\begin{tabular}{|c||c|c|}
\hline Corpus & $\begin{array}{c}\text { Human only } \\
\text { (Eparl+NC) }\end{array}$ & $\begin{array}{c}\text { All WMT'14 } \\
\text { (Eparl+NC+CC) }\end{array}$ \\
\hline \#sents & $2.1 \mathrm{M}$ & $4.5 \mathrm{M}$ \\
BLEU & 21.87 & 24.75 \\
\hline
\end{tabular}

Table 4: Our baseline results on WMT'14 en-de.

post-processing scheme. In particular, we lowercase all data and use a 40k BPE vocabulary (Sennrich et al., 2016b). Before scoring, the case of the hypothesis is restored using a recaser trained on the WMT German news data. Table 4 gives our baseline results using the provided data as it is. We distinguish results when training on human labeled data only, i.e. Europarl and News Commentary (2.1M sentences), and with all WMT'14 training data, i.e. human + Common Crawl (total of 4.5M sentences). Gehring et al. (2017) report a tokenized BLEU score of 25.16 on a slightly different version of newstest-2014 as defined in (Luong et al., 2015). ${ }^{4}$ Please remember that the goal of this paper is not to set a new state-of-the-art in NMT on this data set, but to show relative improvement with respect to a competitive baseline.

\subsection{Filtering Common Crawl}

The Common Crawl corpus is provided by the organizers of WMT'14. We do not know how this corpus was produced, but like all crawled corpora, it is inherently noisy. To filter that corpus, we first embed all the sentences into the joint space and calculate the cosine distance between the English source and the provided German translation. We then extract subsets of different sizes as a function of the threshold on this distance.

\begin{tabular}{|c||c|c|c|}
\hline All & Commas & $<50$ words & LID \\
\hline $2399 \mathrm{k}$ & $2144 \mathrm{k}$ & $2071 \mathrm{k}$ & $1935 \mathrm{k}$ \\
\hline
\end{tabular}

Table 5: Pre-processing of the Common Crawl corpus before distance-based filtering.

After some initial experiments, it turned out that some additional steps are needed before calculating the distances (see Table 5): 1) remove sentences with more than 3 commas. Those are indeed often enumerations of names, cities, etc. While such sentences maybe useful to train NMT systems, the multilingual distance is not very reliable to distinguish list of named entities; 2) limit to sentences with less than 50 words; 3 ) perform LID on source and target sentences; These steps

\footnotetext{
${ }^{4}$ This version uses a subset of 2737 out of 3003 sentences.
}

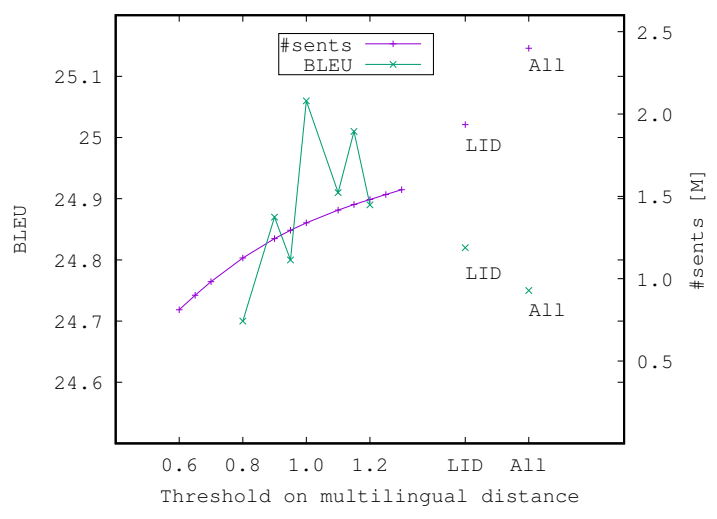

Figure 1: Filtering the Common Crawl corpus: size of corpus (pink) and BLEU scores (green).

discarded overall $19 \%$ of the data. It is surprising that almost $6 \%$ of the data seems to have the wrong source or target language. ${ }^{5}$

Figure 1 (pink curve) shows the amount of data as a function of the threshold on the multilingual distance. Some human inspection of the filtered corpus indicated that the translations start to be wrong for a threshold larger than 1.0. Therefore, we build NMT systems using a filtered version of Common Crawl for thresholds in the range of 0.8 to 1.2 (see Figure 1, green curve). It is good to see that the BLEU score increases when less but better data is used and then decreases again since we discard too much data. Best performance of 25.06 BLEU is achieved for a threshold of 1.0. This corresponds to a gain of 0.3 BLEU on top of a very competitive baseline $(24.75 \rightarrow 25.06)$, using only $3.4 \mathrm{M}$ instead of the original $4.5 \mathrm{M}$ sentence pairs. We actually discard almost half of the Common Crawl data. For comparison, we also trained an NMT system using the pre-processed Common Crawl corpus of $1.9 \mathrm{M}$ sentences (cf. Table 5), but without distance-based filtering. This gives a BLEU score of 24.82, a small 0.07 change.

Aiming at a compromise between speed and full convergence, we trained all systems for 55 epochs which takes less than two days on $8 \mathrm{NVidia}$ GPU100s. Longer training may improve the overall results slightly.

\subsection{Mining Parallel Data in WMT News}

In the framework of the WMT evaluation, large news corpora are provided: 144M English and 187M German sentences (after removing sentence with more than 50 words). As in section 4, we

\footnotetext{
${ }^{5}$ LID itself may also commit errors, we used https: / / fasttext.cc/docs/en/ language-identification.html
} 
embed all sentences into the joint space. For each source sentence, we search for the $k$-nearest sentences in the target language. We use $k=20$ since it can happen that for the same source sentence, several possible translations are found (different news sites reporting on the same fact with different wordings). This search has a complexity of $O(N \times M)$, while filtering presumed parallel corpora is $O(N)$. In our case, $144 M \times 185 M$ amounts to 26 peta distance calculations. This can be quite efficiently done with the highly optimized FAISS toolkit (Johnson et al., 2017).

To start, we trained NMT systems on the extracted data only (see Table 6, 3rd column). As with the Common Crawl corpus, we discarded sentences pairs with the wrong language and many commas. By varying the threshold on the distance between two sentences in the embedding space, we can extract various amounts of data. However, the larger the threshold, the more unlikely the sentences are translations. Training on $1 \mathrm{M}$ mined sentences gives a modest BLEU score of 4.18, which increases up to 7.77 when $4.3 \mathrm{M}$ sentences are extracted. This result is well below an NMT system trained on "real parallel data".

We have observed that the length distribution of the mined sentences is very different of the one of the WMT'14 training corpora (see Figure 2). The average sentence length for all the WMT training corpora is 24 , while it is only 8 words for our mined texts. On one hand, it could be of course that our distance based mining approach works badly for long sentences. But on the other hand, the longer the sentences, the more unlikely it is to find perfect translation in crawled news data. If

\begin{tabular}{|c|c|c|c|c|}
\hline \multirow[b]{2}{*}{ Threshold } & \multirow[b]{2}{*}{ \#Sents } & \multicolumn{3}{|c|}{ BLEU } \\
\hline & & $\begin{array}{l}\text { Mined } \\
\text { alone }\end{array}$ & $\begin{array}{c}\text { Eparl } \\
+ \text { mined }\end{array}$ & $\begin{array}{c}\text { All } \\
+ \text { mined }\end{array}$ \\
\hline baseline & & - & 21.87 & 25.06 \\
\hline 0.25 & $1.0 \mathrm{M}$ & 4.18 & 22.32 & 25.07 \\
\hline 0.26 & $1.5 \mathrm{M}$ & 5.17 & 22.09 & - \\
\hline 0.27 & $1.9 \mathrm{M}$ & 5.92 & 21.97 & - \\
\hline 0.28 & $2.5 \mathrm{M}$ & 6.48 & 22.29 & 25.03 \\
\hline 0.29 & $3.3 \mathrm{M}$ & 6.01 & 22.10 & - \\
\hline 0.30 & $4.3 \mathrm{M}$ & 7.77 & 22.24 & - \\
\hline
\end{tabular}

Table 6: BLEU scores when training on the mined data only, adding it (at different thresholds) to the human translated training corpus (Eparl+NC) and to our best system using filtered Common Crawl.

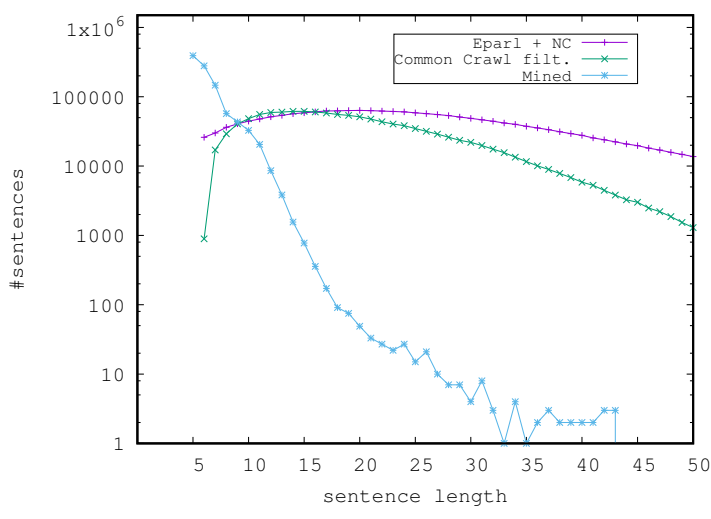

Figure 2: Number of sentences as a function of their length, for WMT'14 training corpora and the mined news texts.

we shuffle the Europarl corpus and consider it as a comparable corpus, our approach is able to extract more than $95 \%$ of the translation pairs. It is also an open question how short sentences impact the training of NMT systems. Further research in those directions is needed.

When adding our mined data to the Europarl and News Commentary corpora (2.1M sentences), we are able to achieve an improvement of 0.45 BLEU $(21.87 \rightarrow 22.32$, 4th column of Table 6$)$. However, we observe no improvement when adding the mined data to our best system which uses the filtered Common Crawl data (5th column of Table 6). It could be that some of our mined data is actually a subset of Common Crawl.

\section{Conclusion}

We have shown that a simple cosine distance in a joint multilingual sentence embedding space can be used to filter noisy parallel data and to mine for bitexts in large news collections. We were able to improve a competitive baseline on the WMT'14 English to German task by 0.3 BLEU by filtering out $25 \%$ of the training data. We will make the filtered and extracted data freely available, as well as a tool to filter noisy bitexts in nine languages.

There are many directions to extend this research, in particular to scale-up to larger corpora. We will apply it to the data mined by the European ParaCrawl project. ${ }^{6}$ The proposed multilingual sentence distance could be also used in MT confidence estimation, or to filter back-translations of monolingual data (Sennrich et al., 2016a).

\footnotetext{
${ }^{6}$ http://paracrawl.eu/download.html
} 


\section{References}

Ashish Vaswani et al. 2017. Attention is all you need. In NIPS.

Amittai Axelrod, Xiaodong He, and Jianfeng Gao. 2011. Domain adaptation via pseudo in-domain data selection. In EMNLP, pages 355-362.

Andoni Azpeitia, Thierry Etchegoyhen, and Eva Martínez Garcia. 2018. Extracting parallel sentences from comparable corpora with STACC variants. In $B U C C$.

Yonatan Belinkov and Yonatan Bisk. 2017. Synthetic and artificial noise both break neural machine translation. In https://arxiv.org/abs/1711. 02173.

Houda Bouamor and Hassan Sajjad. 2018. H2@bucc18: Parallel sentence extraction from comparable coprora using multlingual sentence embeddings. In $B U C C$.

Sarath Chandar, Mitesh M. Khapra, Balaraman Ravindran, Vikas Raykar, and Amrita Saha. 2013. Multilingual deep learning. In NIPS DL wshop.

Junyoung Chunga, Kyunghyun Choa, and Yoshua Bengio. 2016. A character-level decoder without explicit segmentation for neural machine translation. In https://arxiv.org/abs/1603.06147.

Cristina España-Bonet, Ádám Csaba Varga, Alberto Barrón-Cedeño, and Josef van Genabith. 2017. An empirical analysis of nmt-derived interlingual embeddings and their use in parallel sentence identification. IEEE Journal of Selected Topics in Sigmal Processing, pages 1340-1348.

Thierry Etchegoyhen and Andoni Azpeitia. 2016. Settheoretic alignment of comparable corpora. In $A C L$.

Jonas Gehring, Michael Auli, David Grangier, Denis Yarats, and Yann N Dauphin. 2017. Convolutional Sequence to Sequence Learning. In ICML.

Francis Grégoire and Philippe Langlais. 2017. Bucc 2017 shared task: a first attempt toward a deep learning framework for identifying parallel sentences in comparable corpora. In $B U C C$, pages 46-50.

Hany Hassan, Anthony Aue, Chang Chen, Vishal Chowdhary, Jonathan Clark, Christian Federmann, Xuedong Huang, Marcin Junczys-Dowmunt, William Lewis, Mu Li, Shujie Liu, Tie-Yan Liu, Renqian Luo, Arul Menezes, Tao Qin, Frank Seide, $\mathrm{Xu}$ Tan, Fei Tian, Lijun Wu, Shuangzhi Wu, Yingce Xia, Dongdong Zhang, Zhirui Zhang, and Ming Zhou. 2018. Achieving human parity on automatic chinese to english translation. In https: //arxiv.org/abs/1803.05567.

Karl Moritz Hermann and Phil Blunsom. 2014. Multilingual models for compositional distributed semantics. In $A C L$, pages 58-68.
Jeff Johnson, Matthijs Douze, and Hervé Jégou. 2017. Billion-scale similarity search with gpus. arXiv preprint arXiv:1702.08734.

Melvin Johnson et al. 2016. Google's multilingual neural machine translation system: Enabling zero-shot translation. In https: //arxiv.org/ abs/1611.04558.

Chongman Leong, Derek F. Wong, and Lidia S. Chao. 2018. Um-paligner: Neural network-based parallel sentence identification model. In $B U C C$.

Minh-Thang Luong, Hieu Pham, and Christophe D. Manning. 2015. Effective approaches to attentionbased neural machine translation. In $E M N L P$, pages 1412-1421.

Aditua Mogadala and Achim Rettinger. 2016. Bilingual word embeddings from parallel and nonparallel corpora for cross-language classification. In NAACL, pages 692-702.

Dragos Stefan Munteanu and Daniel Marcu. 2005. Improving machine translation performance by exploiting non-parallel corpora. Computational Linguistics, 31(4):477-504.

Hieu Pham, Minh-Thang Luong, and Christopher D. Manning. 2015. Learning distributed representations for multilingual text sequences. In Workshop on Vector Space Modeling for NLP.

Sadaf Abdul Rauf and Holger Schwenk. 2009. On the use of comparable corpora to improve SMT performance. In $E A C L$, pages 16-23.

Philip Resnik and Noah A. Smith. 2003. The web as a parallel corpus. Computational Linguistics, 29:349380.

Lucía Santamaría and Amittai Axelrod. 2017. Data selection with cluster-based language difference models and cynical selection. In IWSLT, pages 137-145.

Holger Schwenk and Matthijs Douze. 2017. Learning joint multilingual sentence representations with neural machine translation. In ACL workshop on Representation Learning for NLP.

Rico Sennrich, Barry Haddow, and Alexandra Birch. 2016a. Improving neural machine translation models with monolingual data. In $A C L$, pages 86-96.

Rico Sennrich, Barry Haddow, and Alexandra Birch. 2016b. Neural machine translation of rare words with subword units. In $A C L$, pages 1715-1725.

Masao Utiyama and Hitoshi Isahara. 2003. Reliable measures for aligning Japanese-English news articles and sentences. In $A C L$, pages 72-79.

Marlies van der Wees, Arianna Bisazza, and Christof Monz. 2017. Dynamic data selection for neural machine translation. In EMNLP, pages 1400-1410. 
Yonghui Wu et al. 2016. Google's neural machine translation system: Bridging the gap between human and machine translation. In https://arxiv. org/abs/1610.05011.

Xinjie Zhou, Xiaojun Wan, and Jianguo Xiao. 2016. Cross-lingual sentiment classification with bilingual document representation learning. In $A C L$.

Pierre Zweigenbaum, Serge Sharoff, and Reinhard Rapp. 2018. Overview of the third bucc shared task: Spottign parallel sentences in comparable corpora. In $B U C C$, pages 60-67. 